\title{
The existence and boundedness of linear and multilinear Marcinkiewicz integrals on central Campanato spaces
}

\author{
Jiao Ma', Mingquan Wei ${ }^{2^{*}}$ (D) and Dunyan Yan'
}

\section{"Correspondence:}

weimingquan11@mails.ucas.ac.cn

${ }^{2}$ School of Mathematics and

Statistics, Xinyang Normal

University, Xinyang, 464000, China

Full list of author information is

available at the end of the article

\section{Springer}

\begin{abstract}
In this paper, we obtain the existence and boundedness of Marcinkiewicz integrals with homogeneous kernels on central Campanato spaces. Moreover, the existence and boundedness of multilinear Marcinkiewicz integrals on central Campanato spaces are also deduced. We extend various previous results to central Campanato spaces which can be seen as the local version of Campanato spaces.
\end{abstract}

Keywords: Marcinkiewicz integral; Homogeneous kernels; Central Campanato spaces

\section{Introduction}

Let $\Omega \in L^{1}\left(\mathbb{S}^{n-1}\right)$ be homogeneous of degree zero and satisfy

$$
\int_{\mathbb{S}^{n-1}} \Omega\left(x^{\prime}\right) d \sigma\left(x^{\prime}\right)=0
$$

where $x^{\prime}=x /|x|$ for any $x \neq 0$. The Marcinkiewicz integral operator of higher dimension is defined by

$$
\mu_{\Omega}(f)(x)=\left(\int_{0}^{\infty}\left|F_{\Omega, t}(x)\right|^{2} \frac{d t}{t^{3}}\right)^{\frac{1}{2}}
$$

where $F_{\Omega, t}(x)=\int_{|x-y| \leq t} \frac{\Omega(x-y)}{\left.|x-y|\right|^{n-1}} f(y) d y$, and $\mathbb{S}^{n-1}$ is the unit sphere of $\mathbb{R}^{n}(n \geq 2)$ equipped with normalized Lebesgue measure $d \sigma=d \sigma\left(x^{\prime}\right)$.

Stein [1] proved that, if $\Omega$ is continuous and satisfies a $\operatorname{Lip}_{\alpha}(0<\alpha \leq 1)$ condition on $\mathbb{S}^{n-1}$, then $\mu_{\Omega}$ is of type $(p, p)(1<p \leq 2)$ and of weak type $(1,1)$. Benedek et al. [2] proved that, if $\Omega \in C^{1}\left(\mathbb{S}^{n-1}\right)$, then $\mu_{\Omega}$ is of type $(p, p)(1<p \leq \infty)$. Ding [3] proved that if $\Omega$ is homogeneous of degree zero satisfying a class of $L^{q}$-Dini $(1<q \leq \infty)$ conditions, and then $\mu_{\Omega}$ is bounded on Campanato spaces.

(c) The Author(s) 2020. This article is licensed under a Creative Commons Attribution 4.0 International License, which permits use, sharing, adaptation, distribution and reproduction in any medium or format, as long as you give appropriate credit to the original author(s) and the source, provide a link to the Creative Commons licence, and indicate if changes were made. The images or other third party material in this article are included in the article's Creative Commons licence, unless indicated otherwise in a credit line to the material. If material is not included in the article's Creative Commons licence and your intended use is not permitted by statutory regulation or exceeds the permitted use, you will need to obtain permission directly from the copyright holder. To view a copy of this licence, visit http://creativecommons.org/licenses/by/4.0/. 
Definition 1.1 Suppose $1 \leq p<\infty$ and $-\frac{n}{p} \leq \alpha<1$. Then the Campanato space $\varepsilon^{\alpha, p}\left(\mathbb{R}^{n}\right)$ is defined as

$$
\varepsilon^{\alpha, p}\left(\mathbb{R}^{n}\right):=\left\{f \in L_{\mathrm{loc}}^{p}\left(\mathbb{R}^{n}\right):\|f\|_{\varepsilon^{\alpha, p}}<\infty\right\}
$$

where

$$
\|f\|_{\varepsilon^{\alpha, p}}=\sup _{x \in \mathbb{R}^{n}, r>0} \frac{1}{|B(x, r)|^{\frac{\alpha}{n}}}\left(\frac{1}{|B(x, r)|} \int_{B(x, r)}\left|f(x)-f_{B(x, r)}\right|^{p} d x\right)^{\frac{1}{p}},
$$

here $B(x, r)=\left\{y \in \mathbb{R}^{n}:|x-y|<r\right\},|B(x, r)|$ is its Lebesgue measure and $f_{B(x, r)}=$ $\frac{1}{|B(x, r)|} \int_{B(x, r)} f(y) d y$.

Remark 1.1 When $\alpha \in(0,1)$ and $p \in[1, \infty), \varepsilon^{\alpha, p}=\operatorname{Lip}_{\alpha}$, where $\operatorname{Lip}_{\alpha}$ is the Lipschitz space; when $\alpha=0$, the Campanato space is the BMO space; when $\alpha \in\left(-\frac{n}{p}, 0\right), \varepsilon^{\alpha, p}=L^{p, \alpha p+n}$, where $L^{p, \alpha p+n}$ is the Morrey space.

Theorem A ([3]) Let $1<p<\infty, 1 \leq q^{\prime} \leq p, \Omega$ satisfy (1.1) and the $L^{q}$-Dini condition. Suppose that $f \in \varepsilon^{\alpha, p}\left(\mathbb{R}^{n}\right)$ for $-\infty<\alpha<0$ and there is a measurable set $E \subset \mathbb{R}^{n}$ with $|E|>0$ such that $\mu_{\Omega}(f)(x)<\infty$ for any $x \in E$. Then $\mu_{\Omega}(f)(x)<\infty$ a.e. on $\mathbb{R}^{n}$ and $\left\|\mu_{\Omega}(f)\right\|_{\varepsilon^{\alpha, p}} \leq$

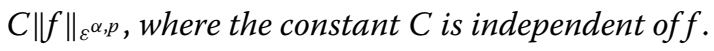

The multilinear theory has attracted much attention since the pioneering work of Coifman and Meyer $[4,5]$. The topic was reconsidered by several authors, including Christ and Journé [6], Kenig and Stein [7], Grafakos and Torres [8, 9], and Lerner et al. [10]. Chen, Xue and Yabuta [11] proved the boundedness of a multilinear Marcinkiewicz integral on Lebesgue spaces. Xue and Yabuta [12] proved the boundedness of a multilinear Marcinkiewicz integral on Campanato spaces.

Definition 1.2 ([11]) Let $\Omega$ be a function defined on $\left(\mathbb{R}^{n}\right)^{m}$ with the following properties:

(1) $\Omega$ is homogeneous of degree 0 , i.e., for any $\lambda>0$ and $\vec{y}=\left(y_{1}, \ldots, y_{m}\right) \in\left(\mathbb{R}^{n}\right)^{m}$,

$$
\Omega(\lambda \vec{y})=\Omega(\vec{y})
$$

(2) $\Omega$ is Lipschitz continuous on $\left(\mathbb{S}^{n-1}\right)^{m}$, i.e. there are $0<\alpha<1$ and $C>0$ such that, for any $\vec{\xi}=\left(\xi_{1}, \ldots, \xi_{m}\right), \vec{\eta}=\left(\eta_{1}, \ldots, \eta_{m}\right) \in\left(\mathbb{R}^{n}\right)^{m}$,

$$
|\Omega(\xi)-\Omega(\eta)| \leq C\left|\xi^{\prime}-\eta^{\prime}\right|^{\alpha}
$$

where $\left(y_{1}, \ldots, y_{m}\right)^{\prime}=\frac{\left(y_{1}, \ldots, y_{m}\right)}{\left|y_{1}\right|+\cdots+\left|y_{m}\right|}$, and we should note that $\left(y_{1}, \ldots, y_{m}\right)^{\prime}$ is not an element of $\left(\mathbb{S}^{n-1}\right)^{m}$;

(3) the integration of $\Omega$ on each unit sphere vanishes,

$$
\int_{\mathbb{S}^{n-1}} \Omega\left(y_{1}, \ldots, y_{m}\right) d y_{j}=0, \quad j=1, \ldots, m
$$


For any $\vec{f}=\left(f_{1}, \ldots, f_{m}\right) \in\left(S\left(\mathbb{R}^{n}\right)\right)^{m}$, we can define the operator $F_{t}$ for any $t>0$ as

$$
\begin{aligned}
F_{t}(\vec{f})(x) & =\frac{\chi(B(0, t))^{m} \Omega(\cdot)}{t^{m}|\vec{\cdot}|^{m(n-1)}} *\left(f_{1} \otimes \cdots \otimes f_{m}\right)(x) \\
& =\frac{1}{t^{m}} \int_{(B(0, t))^{m}} \frac{\Omega(\vec{y})}{|\vec{y}|^{m(n-1)}} \prod_{i=1}^{m} f_{i}\left(x-y_{i}\right) d \vec{y},
\end{aligned}
$$

where $|\vec{y}|=\left|y_{1}\right|+\cdots+\left|y_{m}\right|$ and $B(x, t)=\left\{y \in \mathbb{R}^{n}:|y-x| \leq t\right\}$. Finally, the multilinear Marcinkiewicz integral $\mu$ is defined by

$$
\mu(\vec{f})(x)=\left(\int_{0}^{\infty}\left|F_{t}(\vec{f})(x)\right|^{2} \frac{d t}{t}\right)^{\frac{1}{2}}
$$

If $m=1$, it is easy to see that $\mu(\vec{f})$ coincides with $\mu_{\Omega}(f)$.

Theorem B ([11]) Suppose $\mu$ is bounded from $L^{q_{1}} \times \cdots \times L^{q_{m}}$ to $L^{q}$ for some $1<q_{1}, \ldots, q_{m}<$ $\infty$ with $\frac{1}{q}=\frac{1}{q_{1}}+\cdots+\frac{1}{q_{m}}$. Then, for $1<p_{1}, \ldots, p_{m}<\infty$ with $\frac{1}{p}=\frac{1}{p_{1}}+\cdots+\frac{1}{p_{m}}$, there is $a C>0$ such that

$$
\|\mu(\vec{f})\|_{L^{p}} \leq C \prod_{i=1}^{m}\left\|f_{i}\right\|_{L^{p_{i}}}
$$

Yang [13] introduced the central Campanato spaces on p-adic fields and obtained the behavior of a class of $\mathrm{p}$-adic singular integral operators on these spaces. In fact, the central Campanato space on Euclid spaces can be defined in a similar way. Now we are in a position to define the central Campanato space.

Definition 1.3 ([13]) Suppose $1 \leq p<\infty, q>1, \alpha \in \mathbb{R}$ and $n \in \mathbb{Z}$. The central Campanato space $\mathrm{CL}^{\alpha, p}\left(\mathbb{R}^{n}\right)$ is defined as

$$
\mathrm{CL}^{\alpha, p}\left(\mathbb{R}^{n}\right):=\left\{f \in L_{\mathrm{loc}}^{p}\left(\mathbb{R}^{n}\right):\|f\|_{\mathrm{CL}^{\alpha, p}}<\infty\right\}
$$

where

$$
\|f\|_{C L^{\alpha, p}}=\sup _{r>0} \frac{1}{|B(0, r)|^{\alpha+\frac{1}{p}}}\left(\int_{B(0, r)}\left|f(x)-f_{B(0, r)}\right|^{p} d x\right)^{\frac{1}{p}} .
$$

Remark 1.2 When $\alpha=\frac{1}{l}-1$, the central Campanato space $\mathrm{CL}^{\alpha, p}$ is the central BMO space $\mathrm{CMO}_{l}^{p}$, where $0<l \leq 1<p<\infty$; see [14] for more about the space of $\mathrm{CMO}_{l}^{p}$.

The main results of this paper are as follows.

Theorem 1.1 Let $1<p<\infty, 1 \leq q^{\prime} \leq p, \Omega$ satisfy (1.1) and the $L^{q}$-Dini condition. Suppose that $f \in \mathrm{CL}^{\alpha, p}\left(\mathbb{R}^{n}\right)$ for $-\infty<\alpha n<0$ and there is a measurable set $E \subset \mathbb{R}^{n}$ with $|E|>0$ such that $\mu_{\Omega}(f)(x)<\infty$ for any $x \in E$. Then $\mu_{\Omega}(f)(x)<\infty$ a.e. on $\mathbb{R}^{n}$ and $\left\|\mu_{\Omega}(f)\right\|_{\mathrm{CL}^{\alpha, p}} \leq$ $C\|f\|_{\mathrm{CL}^{\alpha, p}}$, where the constant $C$ is independent off. 
Theorem 1.2 Let $\Omega$ be a function defined on $\left(\mathbb{R}^{n}\right)^{m}$, satisfying (1.2), Lipschitz continuous (1.3) with index replaced by $\beta$ and (1.4). Suppose $\mu$ is bounded from $L^{q_{1}} \times \cdots \times L^{q_{m}}$ to $L^{q}$ for some $1<q_{1}, \ldots, q_{m}<\infty$ with $\frac{1}{q}=\frac{1}{q_{1}}+\cdots+\frac{1}{q_{m}}$. Suppose also that $-\infty<\alpha=\alpha_{1}+\cdots+\alpha_{m}<0$ with $\alpha_{1}, \ldots, \alpha_{m}<0$ and $n<p<\infty$. Then, for $1<p_{1}, \ldots, p_{m}<\infty$ with $\frac{1}{p}=\frac{1}{p_{1}}+\cdots+\frac{1}{p_{m}}$, $f_{j} \in \mathrm{CL}^{\alpha_{j}, p_{j}}\left(\mathbb{R}^{n}\right)(j=1, \ldots, m), \mu(\vec{f})$ is either infinite everywhere or finite almost everywhere, and in the latter case, there is a constant $C>0$ such that

$$
\|\mu(\vec{f})\|_{\mathrm{CL}^{\alpha, p}} \leq C \prod_{j=1}^{m}\left\|f_{j}\right\|_{\mathrm{CL}^{\alpha_{j}, p_{j}}} .
$$

Remark 1.3 Comparing the definition of the Campanato space and the central Campanato space, we can easily see that the selection range of the ball $B$ is different. This leads to the difference in the range of the index about the boundedness of $\mu(\vec{f})$ on Campanato space and central Campanato space.

Throughout this paper we assume that the notation $C$ represents a constant and its values may vary from line to line.

\section{Some preliminaries and notations}

Suppose that $\Omega \in L^{q}\left(\mathbb{S}^{n-1}\right)(q \geq 1)$, the integral modulus $\omega_{q}(\delta)$ of continuity of order $q$ of $\Omega$ is defined by

$$
\omega_{q}(\delta)=\sup _{|\rho| \leq \delta}\left(\int_{\mathbb{S}^{n-1}}\left|\Omega\left(\rho x^{\prime}\right)-\Omega\left(x^{\prime}\right)\right|^{q} d \sigma\left(x^{\prime}\right)\right)^{\frac{1}{q}}
$$

where $\rho$ is a rotation on $\mathbb{S}^{n-1},|\rho|=\|\rho-I\|$. We say that $\Omega$ satisfies the $L^{q}$-Dini condition, if $\omega_{q}(\delta)$ satisfies

$$
\int_{0}^{1} \frac{\omega_{q}(\delta)}{\delta} d \delta<\infty
$$

To prove our main results, we begin with some important lemmas.

Lemma 2.1 ([3]) Suppose that $0<\lambda<n$, and $\Omega$ is homogeneous of degree zero and satisfies the $L^{q}$-Dini condition for $q>1$. If there exists a constant $a_{0}>0$, such that $|x|<a_{0} R$, then we have

$$
\left(\int_{R<|y|<2 R}\left|\frac{\Omega(y-x)}{|y-x|^{n-\lambda}}-\frac{\Omega(y)}{|y|^{n-\lambda}}\right|^{q} d y\right)^{\frac{1}{q}} \leq C R^{\frac{n}{q}-(n-\lambda)}\left(\frac{|x|}{R}+\int_{\frac{|x|}{2 R}<\delta<\frac{|x|}{R}} \frac{\omega_{q}(\delta)}{\delta} d \delta\right),
$$

where the constant $C>0$ is independent of $R$ and $x$.

Lemma 2.2 Suppose that $1<p<\infty,-\infty<\alpha<\frac{1}{2}$, and $\eta>\max \{0, \alpha p n\}$. If $f \in \mathrm{CL}^{\alpha, p}$, then there exists $C>0$ such that, for any ball $B_{0}$ centered at origin with side length $d$ and $x_{0} \in B_{0}$,

$$
\left(\int_{\mathbb{R}^{n}} \frac{d^{\eta}\left|f(x)-f_{B_{0}}\right|^{p}}{d^{n+\eta}+\left|x-x_{0}\right|^{n+\eta}} d x\right)^{\frac{1}{p}} \leq C d^{n \alpha}\|f\|_{\mathrm{CL}^{\alpha, p}} .
$$


Proof Let $B_{k}$ be a ball with the same center of $B_{0}$ and its radius is $2^{k}$ times of $B_{0}$. Decomposing $\mathbb{R}^{n}$ into a geometrically increasing sequence of concentric balls, and using the fact that $\left|f_{B_{k}}-f_{B_{0}}\right| \leq C k\left|B_{k}\right|^{\alpha} \mid f \|_{C L^{\alpha, p}, \text { we have }}$

$$
\begin{aligned}
& \int_{\mathbb{R}^{n}} \frac{d^{\eta}\left|f(x)-f_{B_{0}}\right|^{p}}{d^{n+\eta}+\left|x-x_{0}\right|^{n+\eta}} d x \\
& \quad=\sum_{k=0}^{\infty} \int_{B_{k} \backslash B_{k-1}} \frac{d^{\eta}\left|f(x)-f_{B_{0}}\right|^{p}}{d^{n+\eta}+\left|x-x_{0}\right|^{n+\eta}} d x \\
& \quad \leq C \sum_{k=0}^{\infty} \frac{d^{\eta}}{\left(2^{k} d\right)^{n+\eta}}\left(\int_{B_{k}}\left|f(x)-f_{B_{k}}\right|^{p} d x+\left|f_{B_{k}}-f_{B_{0}}\right|^{p}\left|B_{k}\right|\right) \\
& \quad \leq C \sum_{k=0}^{\infty} \frac{1}{2^{k \eta}}\left(\left(2^{k} d\right)^{n \alpha p}\|f\|_{\mathrm{CL}^{\alpha, p}}^{p}+k^{p}\left(2^{k} d\right)^{n \alpha p} \mid f \|_{\mathrm{CL}^{\alpha, p}}^{p}\right) \\
& \quad \leq C d^{n \alpha p}\|f\|_{\mathrm{CL}^{\alpha, p} .}^{p}
\end{aligned}
$$

Then the proof is complete.

Lemma 2.3 Suppose that $1<p<\infty,-\infty<\alpha<\frac{1}{2}, 1 \leq q^{\prime}<p$ and $\eta>\max \{0, \alpha p n\}$. If $f \in$ $\mathrm{CL}^{\alpha, p}$, then there exists $C>0$ such that, for any ball $B_{0}$ with side length $d$ and centered at origin and $x_{0} \in B_{0}$,

$$
\left(\int_{\mathbb{R}^{n}} \frac{d^{\eta}\left|f(x)-f_{B_{0}}\right|^{q^{\prime}}}{d^{n+\eta}+\left|x-x_{0}\right|^{n+\eta}} d x\right)^{\frac{1}{q^{\prime}}} \leq C d^{n \alpha}\|f\|_{C L^{\alpha, p}}
$$

Proof Let $B_{k}$ be a ball with the same center of $B_{0}$ and its radius is $2^{k}$ times of $B_{0}$. Decomposing $\mathbb{R}^{n}$ into a geometrically increasing sequence of concentric balls, and using Hölder's inequality and Lemma 2.2, we have

$$
\begin{aligned}
& \int_{\mathbb{R}^{n}} \frac{d^{\eta}\left|f(x)-f_{B_{0}}\right|^{q^{\prime}}}{d^{n+\eta}+\left|x-x_{0}\right|^{n+\eta}} d x \\
& \leq \sum_{k=0}^{\infty}\left(\int_{\mathbb{R}^{n}} \frac{d^{\eta}\left|f(x)-f_{B_{0}}\right|^{p}}{d^{n+\eta}+\left|x-x_{0}\right|^{n+\eta}} d x\right)^{\frac{q^{\prime}}{p}} \\
& \quad \times\left(\int_{B_{k} \backslash B_{k-1}} \frac{d^{\eta}}{d^{n+\eta}+\left|x-x_{0}\right|^{n+\eta}} d x\right)^{\frac{p-q^{\prime}}{p}} \\
& \leq C \sum_{k=0}^{\infty} d^{n \alpha q^{\prime}}\|f\|_{\mathrm{CL}}^{q^{\alpha}, p}\left(\frac{d^{\eta}\left(2^{k} d\right)^{n}}{\left(2^{k-1} d\right)^{n+\eta}}\right)^{\frac{p-q^{\prime}}{p}} \\
& \leq C d^{n \alpha q^{\prime}}\|f\|_{\mathrm{CL} L^{\alpha, p} .}^{q^{\prime}}
\end{aligned}
$$

Then the proof is complete. 
Lemma 2.4 Suppose that $1 \leq p<\infty, \alpha<0, B=B(0, r), x \in B$ and $t>8 r>0$. If $f \in \mathrm{CL}^{\alpha, p}$, then, for $0 \leq k \leq k_{0}$ with $k_{0} \in \mathbb{N}$ satisfying $2 r \leq 2^{-k_{0}} t<4 r$, we have

$$
\left(\frac{1}{\left|B\left(x, 2^{-k} t\right)\right|} \int_{B\left(x, 2^{-k} t\right)}\left|f(y)-f_{B}\right|^{p} d y\right)^{\frac{1}{p}} \leq C r^{n \alpha}\|f\|_{\mathrm{CL}^{\alpha, p} .}
$$

Proof Since $x \in B$ and $0 \leq k \leq k_{0}$ satisfying $2 r \leq 2^{-k_{0}} t<4 r$, we have $B\left(x, 2^{-k} t\right) \subset$ $B\left(0,2^{-k+1} t\right)$. Thus

$$
\begin{aligned}
& \left(\frac{1}{\left|B\left(x, 2^{-k} t\right)\right|} \int_{B\left(x, 2^{-k} t\right)}\left|f(y)-f_{B}\right|^{p} d y\right)^{\frac{1}{p}} \\
& \leq C\left(\frac{1}{\left|B\left(0,2^{-k+1} t\right)\right|} \int_{B\left(0,2^{-k+1} t\right)}\left|f(y)-f_{B\left(0,2^{-k+1} t\right)}\right|^{p} d y\right)^{\frac{1}{p}} \\
& \quad+C \sum_{j=k}^{k_{0}}\left|f_{B\left(0,2^{-j+1} t\right)}-f_{B\left(0,2^{-j} t\right)}\right|+C\left|f_{B\left(0,2^{\left.-k_{0} t\right)}\right.}-f_{B}\right| \\
& \leq C\left(2^{-k+1} t\right)^{n \alpha}\left|f \|_{C L^{\alpha, p}}+C \sum_{j=k}^{k_{0}}\right| f_{B\left(0,2^{-j+1} t\right)}-f_{B\left(0,2^{-j} t\right)}|+C| f_{B\left(0,2^{-k_{0}} t\right)}-f_{B} \mid .
\end{aligned}
$$

For $j=k, \ldots, k_{0}$, we have

$$
\begin{aligned}
\left|f_{B\left(0,2^{-j+1} t\right)}-f_{B\left(0,2^{-} t t\right)}\right| & \leq \frac{1}{\left|B\left(0,2^{-j} t\right)\right|} \int_{B\left(0,2^{-j} t\right)}\left|f(y)-f_{B\left(0,2^{-j+1} t\right)}\right| d y \\
& \leq 2^{n}\left(\frac{1}{\left|B\left(0,2^{-j+1} t\right)\right|} \int_{B\left(0,2^{-j+1} t\right)} \mid f(y)-f_{\left.\left.B\left(0,2^{-j+1} t\right)\right|^{p} d y\right)^{\frac{1}{p}}}\right. \\
& \leq 2^{n}\left(2^{-j+1} t\right)^{n \alpha}\|f\|_{\mathrm{CL}^{\alpha, p} .}
\end{aligned}
$$

For the last term, since $2 r \leq 2^{-k_{0}} t \leq 4 r$, we have

$$
\left|f_{B\left(0,2^{-k_{0}} t\right)}-f_{B}\right| \leq 4^{n}\left(2^{-k_{0}} t\right)^{n \alpha}\|f\|_{C L^{\alpha, p}} .
$$

So we obtain

$$
\begin{aligned}
& \left(\frac{1}{\left|B\left(x, 2^{-k} t\right)\right|} \int_{B\left(x, 2^{-k} t\right)}\left|f(y)-f_{B}\right|^{p} d y\right)^{\frac{1}{p}} \\
& \quad \leq C \sum_{j=k}^{k_{0}} 2^{-j n \alpha} t^{n \alpha}\|f\|_{C L^{\alpha, p}} \leq C r^{n \alpha}\|f\|_{C L^{\alpha, p}} .
\end{aligned}
$$

Then the proof is complete.

Lemma 2.5 Suppose that $1 \leq p<\infty, \alpha<0, B=B(0, r), x \in B$ and $k \in \mathbb{N}$. If $\in \mathrm{CL}^{\alpha, p}$, then we have

$$
\left(\frac{1}{\left|B\left(x, 2^{k} r\right)\right|} \int_{B\left(x, 2^{k} r\right)}\left|f(y)-f_{B}\right|^{p} d y\right)^{\frac{1}{p}} \leq C r^{n \alpha}\|f\|_{C L^{\alpha, p} .}
$$


Proof For $x \in B$ and $k \in \mathbb{N}$, we have $B\left(x, 2^{k} r\right) \subset B\left(0,2^{k+1} r\right)$. Similar to Lemma 2.4, we have

$$
\begin{aligned}
& \left(\frac{1}{\left|B\left(x, 2^{k} r\right)\right|} \int_{B\left(x, 2^{k} r\right)}\left|f(y)-f_{B}\right|^{p} d y\right)^{\frac{1}{p}} \\
& \leq C\left(\frac{1}{\left|B\left(0,2^{k+1} r\right)\right|} \int_{B\left(0,2^{k+1} r\right)}\left|f(y)-f_{B\left(0,2^{k+1} r\right)}\right|^{p} d y\right)^{\frac{1}{p}} \\
& \quad+C\left|f_{B\left(0,2^{k+1} r\right)}-f_{B\left(0,2^{k} r\right)}\right|+\cdots+\left|f_{B(0,2 r)}-f_{B}\right| \\
& \leq C \sum_{l=0}^{k+1} 2^{\ln \alpha} r^{n \alpha}\|f\|_{C L^{\alpha, p}} \leq C r^{n \alpha}\|f\|_{C L^{\alpha, p}}
\end{aligned}
$$

Then the proof is complete.

Lemma 2.6 Suppose that $\alpha<0, B=B(0, r), x \in B$ and $t>8 r>0,1 \leq p<\infty$. If $f \in \mathrm{CL}^{\alpha, p}$, then we have

$$
\int_{8 r \leq|x-y|<t} \frac{\left|f(y)-f_{B}\right|}{|x-y|^{n-1}} d y \leq C t r^{n \alpha}\|f\|_{\mathrm{CL}^{\alpha, p}}
$$

Proof Let $k_{0} \in \mathbb{N}$ satisfying $2 r \leq 2^{-k_{0}} t<4 r$. Using Lemma 2.4, we obtain

$$
\begin{aligned}
\int_{8 r \leq|x-y|<t} \frac{\left|f(y)-f_{B}\right|}{|x-y|^{n-1}} d y & \leq \sum_{k=0}^{k_{0}-1} \int_{2^{-k-1} t \leq|x-y| \leq 2^{-k} t} \frac{\left|f(y)-f_{B}\right|}{|x-y|^{n-1}} d y \\
& \leq \sum_{k=0}^{k_{0}-1} \frac{1}{\left(2^{-k-1} t\right)^{n-1}} \int_{B\left(x, 2^{-k} t\right)}\left|f(y)-f_{B}\right| d y \\
& \leq C \sum_{k=0}^{k_{0}-1} 2^{-k} t\left(\frac{1}{\left|B\left(x, 2^{-k} t\right)\right|} \int_{B\left(x, 2^{-k} t\right)}\left|f(y)-f_{B}\right|^{p} d y\right)^{\frac{1}{p}} \\
& \leq C t r^{n \alpha}\|f\|_{C L^{\alpha, p} .}
\end{aligned}
$$

Then the proof is complete.

Lemma 2.7 Suppose that $m \in \mathbb{N}, B=B(0, r), x \in B, 1 \leq p<\infty, 0<\beta \leq 1$ and $\gamma<\beta, \alpha=$ $\alpha_{1}+\cdots+\alpha_{m}<0$ with $\alpha_{1}, \ldots, \alpha_{m}<0$. If $f_{i} \in \mathrm{CL}^{\alpha_{i}, p_{i}}(i=1, \ldots, m)$, then we have

$$
\int_{\left(B(x, 8 r)^{m}\right)^{c}} \frac{r^{\beta} \prod_{i=1}^{m}\left|f_{i}\left(y_{i}\right)-\left(f_{i}\right)_{B}\right|}{\left(\sum_{j=1}^{m}\left|x-y_{j}\right|\right)^{m n+\beta-\gamma}} d \vec{y} \leq C r^{\gamma+n \alpha} \prod_{i=1}^{m}\left\|f_{i}\right\|_{C L^{\alpha}} \alpha_{i}, p_{i} .
$$

Proof Using Lemma 2.5 and Hölder's inequality, we have

$$
\begin{aligned}
& \int_{\left(B(x, 8 r)^{m}\right)^{c}} \frac{r^{\beta} \prod_{i=1}^{m}\left|f_{i}\left(y_{i}\right)-\left(f_{i}\right)_{B}\right|}{\left(\sum_{j=1}^{m}\left|x-y_{j}\right|\right)^{m n+\beta-\gamma}} d \vec{y} \\
& =\sum_{k=0}^{\infty} \int_{\left(B\left(x, 2^{k+4} r\right)\right)^{m} \backslash\left(B\left(x, 2^{k+3} r\right)\right)^{m}} \frac{r^{\beta} \prod_{i=1}^{m}\left|f_{i}\left(y_{i}\right)-\left(f_{i}\right)_{B}\right|}{\left(\sum_{j=1}^{m}\left|x-y_{j}\right|\right)^{m n+\beta-\gamma}} d \vec{y}
\end{aligned}
$$




$$
\begin{aligned}
& \leq C r^{\beta} \sum_{k=0}^{\infty} \frac{1}{\left(2^{k+3} r\right)^{m n+\beta-\gamma}} \int_{\left(B\left(x, 2^{k+4} r\right)^{m}\right.} \prod_{i=1}^{m}\left|f_{i}\left(y_{i}\right)-\left(f_{i}\right)_{B}\right| d \vec{y} \\
& \leq C r^{\gamma} \sum_{k=0}^{\infty} \frac{1}{2^{k(\beta-\gamma)}} \prod_{i=1}^{m} \frac{1}{\left|B\left(x, 2^{k+4} r\right)\right|} \int_{B\left(x, 2^{k+4} r\right)}\left|f_{i}\left(y_{i}\right)-\left(f_{i}\right)_{B}\right| d y_{i} \\
& \leq C \sum_{k=0}^{\infty} \frac{1}{2^{k(\beta-\gamma)}} r^{\gamma+n \alpha} \prod_{i=1}^{m}\left\|f_{i}\right\|_{\mathrm{CL}^{\alpha_{i}, p_{i}}} \\
& \leq C r^{\gamma+n \alpha} \prod_{i=1}^{m}\left\|f_{i}\right\|_{\mathrm{CL}^{\alpha_{i}}, p_{i}} .
\end{aligned}
$$

Then the proof is complete.

\section{Proof the main results}

Proof of Theorem 1.1 Let $1<p<\infty, 1 \leq q^{\prime} \leq p$ and $f \in \mathrm{CL}^{\alpha, p}$. We set $B$ be the ball $B(0, d)$, $B^{*}=8 B=B(0,8 d), B^{j}=2^{j} B=B\left(0,2^{j} d\right)(j \in \mathbb{Z})$. We first show that $\mu_{\Omega}(f)(x)<\infty$, a.e. on $B$.

Let

$$
f(x)=f_{B^{*}}+\left(f(x)-f_{B^{*}}\right) \chi_{B^{*}}(x)+\left(f(x)-f_{B^{*}}\right)\left(1-\chi_{B^{*}}(x)\right)=: f_{1}+f_{2}+f_{3} .
$$

By Theorem A and (1.1), we know that $\mu_{\Omega}\left(f_{1}\right)(x)=0$ on $B$ and

$$
\left(\int_{B}\left|\mu_{\Omega}\left(f_{2}\right)(x)\right|^{p} d x\right)^{\frac{1}{p}} \leq\left(\int_{B^{*}}\left|\mu_{\Omega}\left(f_{2}\right)(x)\right|^{p} d x\right)^{\frac{1}{p}} \leq C d^{n \alpha+\frac{n}{p}}\|f\|_{C L^{\alpha, p}}
$$

Hence

$$
\int_{B}\left|\mu_{\Omega}\left(f_{2}\right)(x)\right| d x \leq|B|^{\frac{1}{p^{\prime}}}\left(\int_{B}\left|\mu_{\Omega}\left(f_{2}\right)(x)\right|^{p} d x\right)^{\frac{1}{p}} \leq d^{n+n \alpha}\|f\|_{C^{\alpha, p}}
$$

This shows that $\mu_{\Omega}\left(f_{2}\right)(x)<\infty$, a.e. on $B$. Since $|E|>0$, we have $|B \cap E|>0$. There exists an $x_{0} \in B \cap E$, such that $\mu_{\Omega}(f)\left(x_{0}\right)<\infty$ and $\mu_{\Omega}\left(f_{2}\right)\left(x_{0}\right)<\infty$. Then

$$
\mu_{\Omega}\left(f_{3}\right)\left(x_{0}\right) \leq \mu_{\Omega}(f)\left(x_{0}\right)+\mu_{\Omega}\left(f_{2}\right)\left(x_{0}\right)<\infty .
$$

Fix any $x \in B$, we write

$$
\begin{aligned}
\left|\mu_{\Omega}\left(f_{3}\right)(x)-\mu_{\Omega}\left(f_{3}\right)\left(x_{0}\right)\right| & \\
\leq & \left(\int_{0}^{\infty}\left(\int_{|x-y|<t,\left|x_{0}-y\right|>t}\left|\frac{\Omega(x-y)}{|x-y|^{n-1}} f_{3}(y)\right| d y\right)^{2} \frac{d t}{t^{3}}\right)^{\frac{1}{2}} \\
& +\left(\int_{0}^{\infty}\left(\int_{|x-y|>t,\left|x_{0}-y\right|<t}\left|\frac{\Omega\left(x_{0}-y\right)}{\left|x_{0}-y\right|^{n-1}} f_{3}(y)\right| d y\right)^{2} \frac{d t}{t^{3}}\right)^{\frac{1}{2}} \\
& +\left(\int_{0}^{\infty}\left(\int_{|x-y|<t,\left|x_{0}-y\right|<t}\left|\frac{\Omega(x-y)}{|x-y|^{n-1}}-\frac{\Omega\left(x_{0}-y\right)}{\left|x_{0}-y\right|^{n-1}}\right|\left|f_{3}(y)\right| d y\right)^{2} \frac{d t}{t^{3}}\right)^{\frac{1}{2}} \\
= & I_{1}+I_{2}+I_{3} .
\end{aligned}
$$


We first estimate $I_{1}$. It is obvious that $|y-x| \approx\left|y-x_{0}\right| \approx|y|$, when $x \in B, x_{0} \in B$ and $y \in\left(B^{*}\right)^{c}$. Applying Minkowski's inequality, we get

$$
\begin{aligned}
I_{1} & \leq \int_{\mathbb{R}^{n}} \frac{|\Omega(x-y)|}{|x-y|^{n-1}}\left|f_{3}(y)\right|\left(\int_{|x-y|<t,\left|x_{0}-y\right|>t} \frac{d t}{t^{3}}\right)^{\frac{1}{2}} d y \\
& \leq C \int_{\left(B^{*}\right)^{c}} \frac{|\Omega(x-y)|}{|x-y|^{n-1}}\left|f_{3}(y)\right|\left|\frac{1}{|x-y|^{2}}-\frac{1}{\left|x_{0}-y\right|^{2}}\right|^{\frac{1}{2}} d y \\
& \leq C \int_{\left(B^{*}\right)^{c}} \frac{|\Omega(x-y)|}{|x-y|^{n-1}}\left|f_{3}(y)\right| \frac{d^{\frac{1}{2}}}{|y|^{\frac{3}{2}}} d y \\
& =C \int_{\left(B^{*}\right)^{c}} \frac{d^{\frac{1}{2}}|\Omega(x-y)|}{|y|^{n+\frac{1}{2}}}\left|f_{3}(y)\right| d y .
\end{aligned}
$$

We take $\eta>0$, such that $\alpha n p<\eta<\frac{q^{\prime}}{2}$. Then $\tau=\left(\frac{1}{2}-\frac{\eta}{q^{\prime}}\right) q>0$. Using Hölder's inequality and Lemma 2.3, we have

$$
\begin{aligned}
I_{1} & \leq C\left(\int_{\left(B^{*}\right)^{c}} \frac{d^{\tau}|\Omega(x-y)|^{q}}{|y|^{n+\tau}} d y\right)^{\frac{1}{q}}\left(\int_{\left(B^{*}\right)^{c}} \frac{d^{\eta}\left|f_{3}(y)\right|^{q^{\prime}}}{|y|^{n+\eta}} d y\right)^{\frac{1}{q^{\prime}}} \\
& \leq C d^{n \alpha}\|f\|_{\mathrm{CL}^{\alpha, p} .}
\end{aligned}
$$

Similarly, we can get $I_{2} \leq C d^{n \alpha}\|f\|_{\mathrm{CL}^{\alpha, p}}$. For the last term $I_{3}$, we use Minkowski's inequality to get

$$
\begin{aligned}
I_{3} & \leq \int_{\mathbb{R}^{n}}\left|\frac{\Omega(x-y)}{|x-y|^{n-1}}-\frac{\Omega\left(x_{0}-y\right)}{\left|x_{0}-y\right|^{n-1}}\right|\left|f_{3}(y)\right|\left(\int_{|x-y|<t,\left|x_{0}-y\right|<t} \frac{d t}{t^{3}}\right)^{\frac{1}{2}} d y \\
& \leq C \int_{\mathbb{R}^{n}}\left|\frac{\Omega(x-y)}{|x-y|^{n-1}}-\frac{\Omega\left(x_{0}-y\right)}{\left|x_{0}-y\right|^{n-1}}\right|\left|f_{3}(y)\right|\left(\int_{\left|x_{0}-y\right|<t}^{\infty} \frac{d t}{t^{3}}\right)^{\frac{1}{2}} d y \\
& \leq C \int_{\mathbb{R}^{n}}\left|\frac{\Omega(x-y)}{|x-y|^{n-1}}-\frac{\Omega\left(x_{0}-y\right)}{\left|x_{0}-y\right|^{n-1}}\right| \frac{\left|f_{3}(y)\right|}{|y|} d y \\
& \leq C \sum_{j=3}^{\infty} \int_{2^{j} d<|y|<2^{j+1} d}\left|\frac{\Omega(x-y)}{|x-y|^{n-1}}-\frac{\Omega\left(x_{0}-y\right)}{\left|x_{0}-y\right|^{n-1}}\right| \frac{\left|f_{3}(y)\right|}{|y|} d y \\
& \leq C \sum_{j=3}^{\infty} \frac{1}{2^{j} d} \int_{2^{j} d<|y|<j^{j+1} d}\left|\frac{\Omega(x-y)}{|x-y|^{n-1}}-\frac{\Omega\left(x_{0}-y\right)}{\left|x_{0}-y\right|^{n-1}}\right|\left|f_{3}(y)\right| d y .
\end{aligned}
$$

Applying Hölder's inequality and Lemma 2.2, we have

$$
\begin{aligned}
I_{3} \leq & C \sum_{j=3}^{\infty} \frac{1}{2^{j} d}\left(\int_{2^{j} d<|y|<2^{j+1} d}\left|\frac{\Omega(x-y)}{|x-y|^{n-1}}-\frac{\Omega\left(x_{0}-y\right)}{\left|x_{0}-y\right|^{n-1}}\right|^{q} d y\right)^{\frac{1}{q}} \\
& \times\left(\int_{2^{j} d<|y|<2^{j+1} d}\left|f_{3}(y)\right|^{q^{\prime}} d y\right)^{\frac{1}{q^{\prime}}} \\
\leq & C \sum_{j=3}^{\infty} \frac{1}{2^{j}}\left(\frac{1}{\left(2^{j+1} d\right)^{n}} \int_{2^{j} d<|y|<2^{j+1} d}\left|f_{3}(y)\right|^{q^{\prime}} d y\right)^{\frac{1}{q^{\prime}}}
\end{aligned}
$$


Ma et al. Journal of Inequalities and Applications

(2020) 2020:194

Page 10 of 18

$$
\begin{aligned}
& +C \sum_{j=3}^{\infty}\left(\int_{\frac{\left|x-x_{0}\right|}{2^{j+1} d}}^{\frac{\left|x-x_{0}\right|}{2^{j} d}} \frac{\omega_{q}(\delta)}{\delta} d \delta\right)\left(\frac{1}{\left(2^{j+1} d\right)^{n}} \int_{2^{j} d<|y|<j^{j+1} d}\left|f_{3}(y)\right|^{q^{\prime}} d y\right)^{\frac{1}{q^{\prime}}} \\
= & I_{31}+I_{32} .
\end{aligned}
$$

To estimate $I_{31}$, we take $\eta>0$. Using Lemma 2.3, it yields

$$
\begin{aligned}
I_{31} & \leq C \sum_{j=3}^{\infty} \frac{1}{2^{j}}\left(\int_{2^{j} d<|y|<2^{j+1} d} \frac{\left(2^{j+1} d\right)^{\eta}\left|f_{3}(y)\right|^{q^{\prime}}}{|y|^{n+\eta}+\left(2^{j+1} d\right)^{n+\eta}} d y\right)^{\frac{1}{q^{\prime}}} \\
& \leq C \sum_{j=3}^{\infty} \frac{1}{2^{j}}\left(\left(\int_{2^{j} d<|y|<2^{j+1} d} \frac{\left(2^{j+1} d\right)^{\eta}\left|f(y)-f_{B_{j}}\right|^{q^{\prime}}}{|y|^{n+\eta}+\left(2^{j+1} d\right)^{n+\eta}} d y\right)^{\frac{1}{q^{\prime}}}+\left|f_{B_{j}}-f_{B^{*}}\right|\right) \\
& \leq C \sum_{j=3}^{\infty} \frac{j}{2^{j}}\left(2^{j} d\right)^{n \alpha}\|f\|_{\mathrm{CL}^{\alpha, p}} \\
& \leq C d^{n \alpha}\|f\|_{\mathrm{CL}^{\alpha, p} .}
\end{aligned}
$$

As for $I_{32}$, we have

$$
\begin{aligned}
I_{32}= & C \sum_{j=3}^{\infty}\left(\int_{\frac{\left|x-x_{0}\right|}{2^{j+1} d}}^{\frac{\left|x-x_{0}\right|}{2^{j} d}} \frac{\omega_{q}(\delta)}{\delta} d \delta\right)\left(\frac{1}{\left(2^{j+1} d\right)^{n}} \int_{2^{j} d<|y|<2^{j+1} d}\left|f(y)-f_{B^{*}}\right|^{q^{\prime}} d y\right)^{\frac{1}{q^{\prime}}} \\
\leq & C \sum_{j=3}^{\infty}\left(\int_{0}^{1} \frac{\omega_{q}(\delta)}{\delta} d \delta\right)\left(\frac{1}{\left(2^{j+1} d\right)^{n}} \int_{2^{j} d<|y|<2^{j+1} d}\left|f(y)-f_{B^{*}}\right|^{q^{\prime}} d y\right)^{\frac{1}{q^{\prime}}} \\
\leq & C \sum_{j=3}^{\infty}\left(\frac{1}{\left(2^{j+1} d\right)^{n}} \int_{2^{j} d<|y|<2^{j+1} d}\left|f(y)-f_{B_{j+1}}\right|^{q^{\prime}} d y\right)^{\frac{1}{q^{\prime}}} \\
& +C \sum_{j=3}^{\infty}\left|f_{B_{j+1}}-f_{B^{*}}\right| \\
= & I_{32}^{1}+I_{32}^{2} .
\end{aligned}
$$

Since $q^{\prime} \leq p$ and using Hölder's inequality, we obtain

$$
\begin{aligned}
I_{32}^{1} & \leq C \sum_{j=3}^{\infty}\left(\frac{1}{\left|B_{j+1}\right|} \int_{B_{j+1}}\left|f(y)-f_{B_{j+1}}\right|^{p} d y\right)^{\frac{1}{p}} \\
& \leq \sum_{j=3}^{\infty}\left|B_{j+1}\right|^{\alpha}\|f\|_{\mathrm{CL}^{\alpha, p}} \\
& \leq C d^{n \alpha}\|f\|_{\mathrm{CL}^{\alpha, p}} .
\end{aligned}
$$

For the last term $I_{32}^{2}$, it is easy to get

$$
\begin{aligned}
I_{32}^{2} & \leq C \sum_{j=3}^{\infty}(j+1)\left(2^{j+1} d\right)^{n \alpha}\|f\|_{\mathrm{CL}^{\alpha, p}} \\
& \leq C d^{n \alpha}\|f\|_{C L^{\alpha, p}} .
\end{aligned}
$$


Summarizing the above estimate, we conclude that

$$
\left|\mu_{\Omega}\left(f_{3}\right)(x)-\mu_{\Omega}\left(f_{3}\right)\left(x_{0}\right)\right| \leq C d^{n \alpha}\|f\|_{C^{\alpha}}{ }^{\alpha, p} .
$$

Thus we have

$$
\begin{aligned}
\mu_{\Omega}(f)(x) \leq & \mu_{\Omega}\left(f_{1}\right)(x)+\mu_{\Omega}\left(f_{2}\right)(x) \\
& +\left|\mu_{\Omega}\left(f_{3}\right)(x)-\mu_{\Omega}\left(f_{3}\right)\left(x_{0}\right)\right|+\mu_{\Omega}\left(f_{3}\right)\left(x_{0}\right)<\infty, \quad \text { a.e. on } B .
\end{aligned}
$$

Because $B$ is any ball centered at the origin, we get $\mu_{\Omega}(f)(x)<\infty$, a.e. on $\mathbb{R}^{n}$. Finally, we show that $\left\|\mu_{\Omega}(f)\right\|_{\mathrm{CL}^{\alpha, p}} \leq C\|f\|_{\mathrm{CL}^{\alpha, p}}$. In fact, from the above proof we find that there exists an $x_{0} \in B$, such that $\mu_{\Omega}\left(f_{3}\right)\left(x_{0}\right)<\infty$. Repeating the above proof, we obtain

$$
\begin{aligned}
& \left(\int_{B}\left|\mu_{\Omega} f(x)-\mu_{\Omega} f_{3}\left(x_{0}\right)\right|^{p} d x\right)^{\frac{1}{p}} \\
& \quad \leq C\left(\int_{B}\left|\mu_{\Omega} f_{2}(x)\right|^{p} d x\right)^{\frac{1}{p}}+\left(\int_{B}\left|\mu_{\Omega} f_{3}(x)-\mu_{\Omega} f_{3}\left(x_{0}\right)\right|^{p} d x\right)^{\frac{1}{p}} \\
& \quad \leq C d^{n \alpha+\frac{n}{p}}\|f\|_{C L^{\alpha, p}} .
\end{aligned}
$$

Taking the supremum over all such $B$, the proof is complete.

Now we give the proof of Theorem 1.2.

Proof of Theorem 1.2 It suffices to verify that, for any $f_{j} \in \mathrm{CL}^{\alpha_{j}, p_{j}}\left(\mathbb{R}^{n}\right)(j=1, \ldots, m)$, if there exists $y_{0} \in \mathbb{R}^{n}$ such that $\mu(\vec{f})\left(y_{0}\right)<\infty$, then, for any ball $B=B(0, r) \subset \mathbb{R}^{n}$ with $y_{0} \in B$,

$$
\left(\frac{1}{|B|} \int_{B}\left|\mu(\vec{f})(x)-(\mu(\vec{f}))_{B}\right|^{p} d x\right)^{\frac{1}{p}} \leq C|B|^{\alpha} \prod_{j=1}^{m}\left\|f_{j}\right\|_{\mathrm{CL}^{\alpha}{ }_{j}, p_{j}} .
$$

For any $r>0$, we denote

$$
\mu^{r}(\vec{f})(x)=\left(\int_{0}^{8 r}\left|\frac{1}{t^{m}} \int_{(B(0, t))^{m}} \frac{\Omega(\vec{y})}{|\vec{y}|^{m(n-1)}} \prod_{i=1}^{m} f_{i}\left(x-y_{i}\right) d \vec{y}\right|^{2} \frac{d t}{t}\right)^{\frac{1}{2}}
$$

and

$$
\mu^{\infty}(\vec{f})(x)=\left(\int_{8 r}^{\infty}\left|\frac{1}{t^{m}} \int_{(B(0, t))^{m}} \frac{\Omega(\vec{y})}{|\vec{y}|^{m(n-1)}} \prod_{i=1}^{m} f_{i}\left(x-y_{i}\right) d \vec{y}\right|^{2} \frac{d t}{t}\right)^{\frac{1}{2}} .
$$

Since $\Omega$ satisfies the vanishing condition (1.4), for any $x \in B$,

$$
\begin{aligned}
\mu^{r}(\vec{f})(x) & =\mu^{r}\left(\left(f_{1}-\left(f_{1}\right)_{B}\right) \chi_{10 B}, \ldots,\left(f_{m}-\left(f_{m}\right)_{B}\right) \chi_{10 B}\right)(x) \\
& \leq \mu\left(\left(f_{1}-\left(f_{1}\right)_{B}\right) \chi_{10 B}, \ldots,\left(f_{m}-\left(f_{m}\right)_{B}\right) \chi_{10 B}\right)(x) .
\end{aligned}
$$


We have $\alpha_{1}+\cdots+\alpha_{m}=\alpha$ and $\frac{1}{p}=\frac{1}{p_{1}}+\cdots+\frac{1}{p_{m}}$ with $1<p_{1}, \ldots, p_{m}<\infty$. Using Theorem B, we get

$$
\begin{aligned}
\left(\int_{B}\left|\mu^{r}(\vec{f})(x)\right|^{p} d x\right)^{\frac{1}{p}} & \leq C \prod_{j=1}^{m}\left(\int_{10 B}\left|f_{j}\left(y_{j}\right)-\left(f_{j}\right)_{10 B}\right|^{p_{j}} d y_{j}\right)^{\frac{1}{p_{j}}} \\
& \leq C \prod_{j=1}^{m}|10 B|^{\alpha_{j}+\frac{1}{p_{j}}}\left\|f_{j}\right\|_{\mathrm{CL}} \alpha_{j j} p_{j} \\
& \leq C|B|^{\alpha+\frac{1}{p}} \prod_{j=1}^{m}\left\|f_{j}\right\|_{\mathrm{CL}} \alpha_{j ; p_{j}} .
\end{aligned}
$$

We notice that

$$
\begin{aligned}
& \left(\frac{1}{|B|} \int_{B}\left|\mu(\vec{f})(x)-(\mu(\vec{f}))_{B}\right|^{p} d x\right)^{\frac{1}{p}} \\
& \quad \leq C\left(\frac{1}{|B|} \int_{B}\left|\mu(\vec{f})(x)-\inf _{y \in B} \mu(\vec{f})(y)\right|^{p} d x\right)^{\frac{1}{p}} \\
& \quad \leq C\left(\frac{1}{|B|} \int_{B}\left|\mu^{r}(\vec{f})(x)\right|^{p} d x\right)^{\frac{1}{p}}+\left(\frac{1}{|B|} \int_{B} \sup _{y \in B}\left|\mu^{\infty}(\vec{f})(x)-\mu^{\infty}(\vec{f})(y)\right|^{p} d x\right)^{\frac{1}{p}} \\
& \quad \leq C|B|^{\alpha} \prod_{j=1}^{m}|| f_{j} \|_{\mathrm{CL}^{\alpha}{ }_{j}, p_{j}}+\left(\frac{1}{|B|} \int_{B} \sup _{y \in B}\left|\mu^{\infty}(\vec{f})(x)-\mu^{\infty}(\vec{f})(y)\right|^{p} d x\right)^{\frac{1}{p}} .
\end{aligned}
$$

So the proof of Theorem 1.2 reduces to proving that, for any $x, z \in B$,

$$
\left|\mu^{\infty}(\vec{f})(x)-\mu^{\infty}(\vec{f})(z)\right| \leq C|B|^{\alpha} \prod_{j=1}^{m}\left\|f_{j}\right\|_{\mathrm{CL}^{\alpha_{j}, p_{j}}}
$$

It is easy to see that

$$
\begin{aligned}
\left|\mu^{\infty}(\vec{f})(x)-\mu^{\infty}(\vec{f})(z)\right| & =\left|\left(\int_{8 r}^{\infty}\left|F_{t}(\vec{f})(x)\right|^{2} \frac{d t}{t}\right)^{\frac{1}{2}}-\left(\int_{8 r}^{\infty}\left|F_{t}(\vec{f})(z)\right|^{2} \frac{d t}{t}\right)^{\frac{1}{2}}\right| \\
& \leq\left(\int_{8 r}^{\infty}\left|F_{t}(\vec{f})(x)-F_{t}(\vec{f})(z)\right|\left|F_{t}(\vec{f})(x)+F_{t}(\vec{f})(z)\right| \frac{d t}{t}\right)^{\frac{1}{2}} .
\end{aligned}
$$

For any $z \in B, t_{1}, \ldots, t_{m}>r$, we have $B\left(z, t_{i}\right) \subset B\left(0,2 t_{i}\right)(i=1, \ldots, m)$. If $n<p<\infty$ and $-\infty<$ $\alpha<0$, the vanishing condition of $\Omega$ and Hölder's inequality allow us to obtain

$$
\begin{gathered}
\left|\int_{\prod_{i=1}^{m} B\left(z, t_{i}\right)} \frac{\Omega\left(z-y_{1}, \ldots, z-y_{m}\right)}{\left(\sum_{j=1}^{m}\left|z-y_{j}\right|\right)^{m(n-1)}} \prod_{i=1}^{m} f_{i}\left(y_{i}\right) d \vec{y}\right| \\
\leq C\left(\int_{\prod_{i=1}^{m} B\left(z, t_{i}\right)}\left|\prod_{j=1}^{m}\left(f_{j}\left(y_{j}\right)-\left(f_{j}\right)_{B\left(0,2 t_{j}\right)}\right)\right|^{p} d \vec{y}\right)^{\frac{1}{p}} \\
\quad \times\left(\int_{\prod_{i=1}^{m} B\left(z, t_{i}\right)} \frac{d \vec{y}}{\left(\sum_{j=1}^{m}\left|z-y_{j}\right|\right)^{m(n-1) p^{\prime}}}\right)^{\frac{1}{p^{\prime}}}
\end{gathered}
$$




$$
\begin{aligned}
\leq & C\left(\int_{\prod_{i=1}^{m} B\left(0,2 t_{i}\right)}\left|\prod_{j=1}^{m}\left(f_{j}\left(y_{j}\right)-\left(f_{j}\right)_{B\left(0,2 t_{j}\right.}\right)\right|^{p} d \vec{y}\right)^{\frac{1}{p}} \\
& \times\left(\int_{\prod_{i=1}^{m} B\left(z, t_{i}\right)} \frac{d \vec{y}}{\left(\sum_{j=1}^{m}\left|z-y_{j}\right|\right)^{m(n-1) p^{\prime}}}\right)^{\frac{1}{p^{\prime}}} \\
\leq & C \prod_{j=1}^{m} t_{j}^{\frac{n}{p^{p^{\prime}}}-n+1} \prod_{j=1}^{m}\left(\prod_{i \neq j} t_{i}^{\frac{n}{p_{j}}} t_{j}^{\frac{n}{p_{j}}+n \alpha_{j}}\right)\left\|f_{j}\right\|_{\mathrm{CL}} \alpha_{j, p_{j}} \\
= & C \prod_{j=1}^{m} t_{j}^{\frac{n}{p^{\prime}}-n+1} \prod_{j=1}^{m} t_{j}^{\frac{n}{p}+n \alpha_{j}}\left\|f_{j}\right\|_{\mathrm{CL}^{\alpha_{j}, p_{j}}} \\
= & C \prod_{j=1}^{m} t_{j}^{1+n \alpha_{j}}\left\|f_{j}\right\|_{\mathrm{CL}^{\alpha_{j}, p_{j}} .}
\end{aligned}
$$

For $z \in B, t>8 r, B(z, t)$ can be decomposed into the following disjoint union:

$$
\begin{aligned}
(B(z, t))^{m}= & \left\{\bigcup_{i=1}^{m}\left((B(z, t) \backslash B(z, 8 r))^{i-1} \times B(z, 8 r) \times(B(z, t) \backslash B(z, 8 r))^{m-i}\right)\right\} \\
& \cup(B(z, t) \backslash B(z, 8 r))^{m} \cup(B(z, 8 r))^{m} .
\end{aligned}
$$

Set $B_{i}(z, t, r):=(B(z, t) \backslash B(z, 8 r))^{i-1} \times B(z, 8 r) \times(B(z, t) \backslash B(z, 8 r))^{m-i}$. We write

$$
\begin{aligned}
t^{m} F_{t}(\vec{f})(z)= & \int_{(B(z, t))^{m}} \frac{\Omega\left(z-y_{1}, \ldots, z-y_{m}\right)}{\left(\sum_{j=1}^{m}\left|z-y_{j}\right|\right)^{m(n-1)}} \prod_{i=1}^{m} f_{i}\left(y_{i}\right) d \vec{y} \\
= & \int_{(B(z, t) \backslash B(z, 8 r))^{m}} \frac{\Omega\left(z-y_{1}, \ldots, z-y_{m}\right)}{\left(\sum_{j=1}^{m}\left|z-y_{j}\right|\right)^{m(n-1)}} \prod_{i=1}^{m} f_{i}\left(y_{i}\right) d \vec{y} \\
& +\sum_{l=1}^{m} \int_{B_{l}(z, t, r)} \frac{\Omega\left(z-y_{1}, \ldots, z-y_{m}\right)}{\left(\sum_{j=1}^{m}\left|z-y_{j}\right|\right)^{m(n-1)}} \prod_{i=1}^{m} f_{i}\left(y_{i}\right) d \vec{y} \\
& +\int_{(B(z, 8 r))^{m}} \frac{\Omega\left(z-y_{1}, \ldots, z-y_{m}\right)}{\left(\sum_{j=1}^{m}\left|z-y_{j}\right|\right)^{m(n-1)}} \prod_{i=1}^{m} f_{i}\left(y_{i}\right) d \vec{y} .
\end{aligned}
$$

According to the above estimate, we have

$$
\begin{aligned}
& \left|t^{m} F_{t}(\vec{f})(z)\right| \leq C t^{m+n \alpha} \prod_{j=1}^{m}\left\|f_{j}\right\|_{\mathrm{CL}^{\alpha_{j}, p_{j}}} ; \\
& \left|\int_{B_{l}(z, t, r)} \frac{\Omega\left(z-y_{1}, \ldots, z-y_{m}\right)}{\left(\sum_{j=1}^{m}\left|z-y_{j}\right|\right)^{m(n-1)}} \prod_{i=1}^{m} f_{i}\left(y_{i}\right) d \vec{y}\right| \\
& \quad \leq C t^{m-1+n\left(\alpha-\alpha_{l}\right)} r^{1+n \alpha_{l}} \prod_{j=1}^{m}\left\|f_{j}\right\|_{\mathrm{CL}^{\alpha_{j}}, p_{j}} ; \\
& \left|\int_{(B(z, 8 r))^{m}} \frac{\Omega\left(z-y_{1}, \ldots, z-y_{m}\right)}{\left(\sum_{j=1}^{m}\left|z-y_{j}\right|\right)^{m(n-1)}} \prod_{i=1}^{m} f_{i}\left(y_{i}\right) d \vec{y}\right| \leq C r^{m+n \alpha} \prod_{j=1}^{m}\left\|f_{j}\right\|_{\mathrm{CL}^{\alpha} p_{j}, p_{j}}
\end{aligned}
$$


For $x, z \in B, t \geq 8 r$, we set

$$
\begin{aligned}
H_{t}(\vec{f})(x, z)=: & \mid \int_{(B(z, t) \backslash B(z, 8 r))^{m}} \frac{\Omega\left(z-y_{1}, \ldots, z-y_{m}\right)}{\left(\sum_{j=1}^{m}\left|z-y_{j}\right|\right)^{m(n-1)}} \prod_{i=1}^{m} f_{i}\left(y_{i}\right) d \vec{y} \\
& -\int_{(B(x, t) \backslash B(x, 8 r))^{m}} \frac{\Omega\left(x-y_{1}, \ldots, x-y_{m}\right)}{\left(\sum_{j=1}^{m}\left|x-y_{j}\right|\right)^{m(n-1)}} \prod_{i=1}^{m} f_{i}\left(y_{i}\right) d \vec{y} \mid .
\end{aligned}
$$

Consequently, we have

$$
\begin{aligned}
& \left|\mu^{\infty}(\vec{f})(x)-\mu^{\infty}(\vec{f})(z)\right| \\
& \leq C\left(\int_{8 r}^{\infty}\left|F_{t}(\vec{f})(x)-F_{t}(\vec{f})(z)\right| \frac{d t}{t^{1-n \alpha}}\right)^{\frac{1}{2}} \prod_{j=1}^{m}\left\|f_{j}\right\|_{\mathrm{CL}^{\frac{\alpha}{j}}, p_{j}}^{\frac{1}{2}} \\
& \leq C \prod_{j=1}^{m}\left\|f_{j}\right\|_{\mathrm{CL}^{\alpha_{j}, p_{j}}}\left(\int_{8 r}^{\infty}\left(\sum_{j=1}^{m} t^{m-1+n\left(\alpha-\alpha_{j}\right)} r^{1+n \alpha_{j}}+r^{m+n \alpha}\right) \frac{d t}{t^{m+1-n \alpha}}\right)^{\frac{1}{2}} \\
& +C \prod_{j=1}^{m}\left\|f_{j}\right\|_{\mathrm{CL}^{\frac{1}{2}}}^{\frac{1}{2}} p_{j}\left(\int_{8 r}^{\infty}\left|H_{t}(\vec{f})(x, z)\right| \frac{d t}{t^{m+1-n \alpha}}\right)^{\frac{1}{2}} \\
& \leq C r^{n \alpha} \prod_{j=1}^{m}\left\|f_{j}\right\|_{\mathrm{CL}^{\alpha} \alpha_{j}, p_{j}}+C \prod_{j=1}^{m}\left\|f_{j}\right\|_{\mathrm{CL}^{2} \alpha_{j}, p_{j}}^{\frac{1}{2}}\left(\int_{8 r}^{\infty}\left|H_{t}(\vec{f})(x, z)\right| \frac{d t}{t^{m+1-n \alpha}}\right)^{\frac{1}{2}} .
\end{aligned}
$$

Fixing $x, z$ and for $t>0$, we introduce some notations:

$$
\begin{aligned}
& \Xi(x, t)=\left\{y \in \mathbb{R}^{n}: 8 r \leq|x-y|<t, 8 r \leq|z-y|<t\right\} \\
& \Xi(z, t)=\left\{y \in \mathbb{R}^{n}: 8 r \leq|z-y|<t, 8 r \leq|x-y|<t\right\} \\
& \Gamma(x, t)=\left\{y \in \mathbb{R}^{n}: 8 r \leq|x-y|<t,|z-y| \geq t\right\} \\
& \Gamma(z, t)=\left\{y \in \mathbb{R}^{n}: 8 r \leq|z-y|<t,|x-y| \geq t\right\} \\
& \Lambda(x, t)=\left\{y \in \mathbb{R}^{n}: 8 r \leq|x-y|<t,|z-y|<8 r\right\} \\
& \Lambda(z, t)=\left\{y \in \mathbb{R}^{n}: 8 r \leq|z-y|<t,|x-y|<8 r\right\} ; \\
& \vec{\Theta}(x, t)=\Theta_{1}(x, t) \times \cdots \times \Theta_{m}(x, t), \Theta_{i}(x, t) \in\{\Xi(x, t), \Gamma(x, t), \Lambda(x, t)\} \\
& \vec{\Theta}(z, t)=\Theta_{1}(z, t) \times \cdots \times \Theta_{m}(z, t), \Theta_{i}(z, t) \in\{\Xi(z, t), \Gamma(z, t), \Lambda(z, t)\} \\
& \Xi(x, t)=\Xi(z, t)=: \Xi(t) .
\end{aligned}
$$

For $y$, we denote

$$
\begin{aligned}
& \Xi(x, y)=\{t>0: 8 r \leq|x-y|<t, 8 r \leq|z-y|<t\} \\
& \Xi(z, y)=\{t>0: 8 r \leq|z-y|<t, 8 r \leq|x-y|<t\} \\
& \Gamma(x, y)=\{t>0: 8 r \leq|x-y|<t,|z-y| \geq t\} \\
& \Gamma(z, y)=\{t>0: 8 r \leq|z-y|<t,|x-y| \geq t\} \\
& \Lambda(x, y)=\{t>0: 8 r \leq|x-y|<t,|z-y|<8 r\}
\end{aligned}
$$




$$
\begin{aligned}
& \Lambda(z, y)=\{t>0: 8 r \leq|z-y|<t,|x-y|<8 r\} \\
& \Theta_{i}\left(x, y_{i}\right) \in\left\{\Xi\left(x, y_{i}\right), \Gamma\left(x, y_{i}\right)\right\}, \quad i=1, \ldots, m ; \\
& \Theta_{i}\left(z, y_{i}\right) \in\left\{\Xi\left(z, y_{i}\right), \Gamma\left(z, y_{i}\right)\right\}, \quad i=1, \ldots, m .
\end{aligned}
$$

It is easy to see that

$$
B(x, t) \backslash B(x, 8 r)=\Xi(x, t) \cup \Gamma(x, t) \cup \Lambda(x, t)
$$

and

$$
B(z, t) \backslash B(z, 8 r)=\Xi(z, t) \cup \Gamma(z, t) \cup \Lambda(z, t) .
$$

We write

$$
\begin{aligned}
& H_{t}(\vec{f})(x, z) \leq \int_{(\Xi(t))^{m}}\left|\frac{\Omega\left(x-y_{1}, \ldots, x-y_{m}\right)}{\left(\sum_{j=1}^{m}\left|x-y_{j}\right|\right)^{m(n-1)}}-\frac{\Omega\left(z-y_{1}, \ldots, z-y_{m}\right)}{\left(\sum_{j=1}^{m}\left|z-y_{j}\right|\right)^{m(n-1)}}\right| \\
& \times \prod_{i=1}^{m}\left|f_{i}\left(y_{i}\right)-\left(f_{i}\right)_{B}\right| d \vec{y} \\
& +\int_{(\Lambda(x, t))^{m}} \frac{\left|\Omega\left(x-y_{1}, \ldots, x-y_{m}\right)\right|}{\left(\sum_{j=1}^{m}\left|x-y_{j}\right|\right)^{m(n-1)}} \prod_{i=1}^{m}\left|f_{i}\left(y_{i}\right)-\left(f_{i}\right)_{B}\right| d \vec{y} \\
& +\int_{(\Lambda(z, t))^{m}} \frac{\left|\Omega\left(z-y_{1}, \ldots, z-y_{m}\right)\right|}{\left(\sum_{j=1}^{m}\left|z-y_{j}\right|\right)^{m(n-1)}} \prod_{i=1}^{m}\left|f_{i}\left(y_{i}\right)-\left(f_{i}\right)_{B}\right| d \vec{y} \\
& +\int_{\vec{\Theta}(x, t), \exists \Theta_{i}(x, t)=\Gamma(x, t)} \frac{\left|\Omega\left(x-y_{1}, \ldots, x-y_{m}\right)\right|}{\left(\sum_{j=1}^{m}\left|x-y_{j}\right|\right)^{m(n-1)}} \prod_{i=1}^{m}\left|f_{i}\left(y_{i}\right)-\left(f_{i}\right)_{B}\right| d \vec{y} \\
& +\int_{\vec{\Theta}(z, t), \exists \Theta_{i}(z, t)=\Gamma(z, t)} \frac{\left|\Omega\left(z-y_{1}, \ldots, z-y_{m}\right)\right|}{\left(\sum_{j=1}^{m}\left|z-y_{j}\right|\right)^{m(n-1)}} \prod_{i=1}^{m}\left|f_{i}\left(y_{i}\right)-\left(f_{i}\right)_{B}\right| d \vec{y} \\
& +\sum_{l=1}^{m-1} \int_{(\Xi(x, t))^{l}} \int_{(\Lambda(x, t))^{m-l}} \frac{\left|\Omega\left(x-y_{1}, \ldots, x-y_{m}\right)\right|}{\left(\sum_{j=1}^{m}\left|x-y_{j}\right|\right)^{m(n-1)}} \prod_{i=1}^{m}\left|f_{i}\left(y_{i}\right)-\left(f_{i}\right)_{B}\right| d \vec{y} \\
& +\sum_{l=1}^{m-1} \int_{(\Xi(z, t))^{l}} \int_{(\Lambda(z, t))^{m-l}} \frac{\left|\Omega\left(z-y_{1}, \ldots, z-y_{m}\right)\right|}{\left(\sum_{j=1}^{m}\left|z-y_{j}\right|\right)^{m(n-1)}} \prod_{i=1}^{m}\left|f_{i}\left(y_{i}\right)-\left(f_{i}\right)_{B}\right| d \vec{y} \\
& =: \sum_{i=1}^{5} H_{t, i}(\vec{f})(x, z)+\sum_{l=1}^{m-1} H_{t, 6}^{l}(\vec{f})(x, z)+\sum_{l=1}^{m-1} H_{t, 7}^{l}(\vec{f})(x, z) \text {. }
\end{aligned}
$$

For $x, z \in B$ and $\Omega$ satisfying Lipschitz continuous condition, applying Lemma 2.7, we get

$$
\begin{aligned}
& \int_{8 r}^{\infty}\left|H_{t, 1}(\vec{f})(x, z)\right| \frac{d t}{t^{m+1-n \alpha}} \\
& \quad \leq C \int_{\left((B(x, 8 r))^{c}\right)^{m}} \frac{|x-z|^{\beta}}{\left(\sum_{j=1}^{m}\left|x-y_{j}\right|\right)^{m(n-1)+\beta}}
\end{aligned}
$$




$$
\begin{aligned}
& \times \prod_{i=1}^{m}\left|f_{i}\left(y_{i}\right)-\left(f_{i}\right)_{B}\right| d \vec{y} \int_{\frac{1}{m}\left(\sum_{j=1}^{m}\left|x-y_{j}\right|\right)} \frac{d t}{t^{m+1-n \alpha}} d \vec{y} \\
\leq & C \int_{\left((B(x, 8 r))^{c}\right)^{m}} \frac{r^{\beta} \prod_{i=1}^{m}\left|f_{i}\left(y_{i}\right)-\left(f_{i}\right)_{B}\right|}{\left(\sum_{j=1}^{m}\left|x-y_{j}\right|\right)^{m n+\beta-n \alpha}} d \vec{y} \\
\leq & C r^{2 n \alpha} \prod_{j=1}^{m}\left\|f_{j}\right\|_{\mathrm{CL}^{\alpha}{ }^{\alpha}, p_{j}} .
\end{aligned}
$$

When $x, z \in B, 8 r \leq|x-y|<t$ and $|z-y|<8 r$, we have $|x-y|<|x-z|+|z-y|<2 r+8 r=$ $10 r$. Then $8 r<|x-y|<10 r$. Applying Lemma 2.6, we get

$$
\begin{aligned}
\left|H_{t, 2}(\vec{f})(x, z)\right| & \leq C \int_{(B(x, 10 r) \backslash B(x, 8 r))^{m}} \frac{\prod_{i=1}^{m}\left|f_{i}\left(y_{i}\right)-\left(f_{i}\right)_{B}\right|}{\left(\sum_{j=1}^{m}\left|x-y_{j}\right|\right)^{m(n-1)}} d \vec{y} \\
& \leq \prod_{i=1}^{m} \int_{8 r \leq\left|x-y_{i}\right| \leq 10 r} \frac{\left|f_{i}\left(y_{i}\right)-\left(f_{i}\right)_{B}\right|}{\left|x-y_{i}\right|^{n-1}} d y_{i} \\
& \leq C r^{m+n \alpha} \prod_{j=1}^{m}\left\|f_{j}\right\|_{\mathrm{CL}^{\alpha_{j}, p_{j}}},
\end{aligned}
$$

which leads to

$$
\int_{8 r}^{\infty}\left|H_{t, 2}(\vec{f})(x, z)\right| \frac{d t}{t^{m+1-n \alpha}} \leq C r^{2 n \alpha} \prod_{j=1}^{m}\left\|f_{j}\right\|_{\mathrm{CL}^{\alpha_{j}, p_{j}}}
$$

Similarly,

$$
\int_{8 r}^{\infty}\left|H_{t, 3}(\vec{f})(x, z)\right| \frac{d t}{t^{m+1-n \alpha}} \leq C r^{2 n \alpha} \prod_{j=1}^{m}\left\|f_{j}\right\|_{\mathrm{CL}^{\alpha}{ }^{\alpha}, p_{j}}
$$

Now we estimate $H_{t, 4}(\vec{f})$. For any $x, z \in B$,

$$
\left|\bigcap_{j=1}^{m} \Theta_{j}\left(x, y_{j}\right)\right| \leq\left|\Theta_{i}\left(x, y_{i}\right)\right|=\left|\Gamma\left(x, y_{i}\right)\right| \leq|| z-y_{i}|-| x-y_{i}|| \leq|z-x| \leq 2 r .
$$

And for any $t \in \bigcap_{j=1}^{m} \Theta_{j}\left(x, y_{j}\right), t>\frac{1}{m}\left(\sum_{j=1}^{m}\left|x-y_{j}\right|\right)$. Applying Lemma 2.7, we get

$$
\begin{aligned}
& \int_{8 r}^{\infty}\left|H_{t, 4}(\vec{f})(x, z)\right| \frac{d t}{t^{m+1-n \alpha}} \\
& \leq C \int_{\left((B(x, 8 r))^{c}\right)^{m}} \frac{\prod_{i=1}^{m}\left|f_{i}\left(y_{i}\right)-\left(f_{i}\right)_{B}\right|}{\left(\sum_{j=1}^{m}\left|x-y_{j}\right|\right)^{m(n-1)}} d \vec{y} \\
& \quad \times \int_{\bigcap_{j=1}^{m} \Theta_{j}\left(x, y_{j}\right)} \frac{d t}{t^{m+1-n \alpha}} \\
& \leq C r \int_{\left((B(x, 8 r))^{c}\right)^{m}} \frac{\prod_{i=1}^{m}\left|f_{i}\left(y_{i}\right)-\left(f_{i}\right)_{B}\right|}{\left(\sum_{j=1}^{m}\left|x-y_{j}\right|\right)^{m n+1-n \alpha}} d \vec{y} \\
& \leq C r^{2 n \alpha} \prod_{j=1}^{m}\left\|f_{j}\right\|_{\mathrm{CL}^{\alpha_{j}, p_{j}} .}
\end{aligned}
$$


Similarly,

$$
\int_{8 r}^{\infty}\left|H_{t, 5}(\vec{f})(x, z)\right| \frac{d t}{t^{m+1-n \alpha}} \leq C r^{2 n \alpha} \prod_{j=1}^{m}\left\|f_{j}\right\|_{\mathrm{CL}^{\alpha_{j}, p_{j}}} .
$$

As for $H_{t, 6}^{l}(\vec{f})$, suppose $y_{1}, \ldots, y_{l} \in \Xi(t), y_{l+1}, \ldots, y_{m} \in \Lambda(x, t)$. Using Lemma 2.6, we have

$$
\begin{aligned}
H_{t, 6}^{l}(\vec{f})(x, z) \leq & \prod_{j=1}^{l} \int_{8 r \leq\left|x-y_{j}\right|<t} \frac{\left|f_{j}\left(y_{j}\right)-\left(f_{j}\right)_{B}\right|}{\left|x-y_{j}\right|^{n-1}} d y_{j} \\
& \times \prod_{j=l+1}^{m} \int_{8 r \leq\left|x-y_{j}\right| \leq 10 r} \frac{\left|f_{j}\left(y_{j}\right)-\left(f_{j}\right)_{B}\right|}{\left|x-y_{j}\right|^{n-1}} d y_{j} \\
\leq & C \prod_{j=1}^{l}\left(t r^{n \alpha_{j}}\right)\left\|f_{j}\right\|_{\mathrm{CL}^{\alpha_{j}, p_{j}}} \prod_{j=l+1}^{m} r^{1+n \alpha_{j}}\left\|f_{j}\right\|_{\mathrm{CL}^{\alpha_{j}, p_{j}}} \\
= & C t^{l} r^{m-l+n \alpha} \prod_{j=1}^{m}\left\|f_{j}\right\|_{\mathrm{CL}^{\alpha_{j}, p_{j}} .}
\end{aligned}
$$

So we obtain

$$
\begin{aligned}
\int_{8 r}^{\infty}\left|H_{t, 6}^{l}(\vec{f})(x, z)\right| \frac{d t}{t^{m+1-n \alpha}} & \leq C r^{m-l+n \alpha} \int_{8 r}^{\infty} t^{l-m-1+n \alpha} d t \prod_{j=1}^{m}\left\|f_{j}\right\|_{\mathrm{CL}^{\alpha} p_{j} p_{j}} \\
& \leq C r^{2 n \alpha} \prod_{j=1}^{m}\left\|f_{j}\right\|_{\mathrm{CL}^{\alpha_{j}, p_{j}} .}
\end{aligned}
$$

Similarly,

$$
\int_{8 r}^{\infty}\left|H_{t, 7}^{l}(\vec{f})(x, z)\right| \frac{d t}{t^{m+1-n \alpha}} \leq C r^{2 n \alpha} \prod_{j=1}^{m}\left\|f_{j}\right\|_{\mathrm{CL}^{\alpha_{j}, p_{j}}}
$$

Combining the above estimates, we have

$$
\left(\int_{8 r}^{\infty}\left|H_{t}(\vec{f})(x, z)\right| \frac{d t}{t^{m+1-n \alpha}}\right)^{\frac{1}{2}} \leq C r^{n \alpha} \prod_{j=1}^{m}\left\|f_{j}\right\|_{\mathrm{CL}^{\frac{1}{2}} p_{j},}^{\frac{1}{2}} .
$$

Consequently, we have

$$
\left|\mu^{\infty}(\vec{f})(x)-\mu^{\infty}(\vec{f})(z)\right| \leq C r^{n \alpha} \prod_{j=1}^{m}\left\|f_{j}\right\|_{\mathrm{CL}^{\alpha_{j}, p_{j}}} .
$$

Then the proof is complete. 
Funding

This work is supported by the National Natural Science Foundation of China (No. 11871452), and Project of Henan Provincial Department of Education (18A110028) and the Nanhu Scholar Program for Young Scholars of Xinyang Normal University.

Availability of data and materials

Not applicable.

Competing interests

The authors declare that they have no competing interests.

\section{Authors' contributions}

All authors contributed equally to the writing of this paper. All authors read and approved the final manuscript.

\section{Author details}

${ }^{1}$ School of Mathematics, University of Chinese Academy of Sciences, Beijing, 100049, China. ${ }^{2}$ School of Mathematics and Statistics, Xinyang Normal University, Xinyang, 464000, China.

\section{Publisher's Note}

Springer Nature remains neutral with regard to jurisdictional claims in published maps and institutional affiliations.

Received: 28 April 2020 Accepted: 23 July 2020 Published online: 29 July 2020

\section{References}

1. Stein, E.: On the function of Littlewood-Paley, Lusin and Marcinkiewicz. Transl. Am. Math. Soc. 88, 430-466 (1958)

2. Benedek, A., Caldron, A., Panzone, R.: Convolution operators on Banach space valued functions. Proc. Natl. Acad. Sci. USA 48, 356-365 (1962)

3. Ding, Y., Lu, S., Xue, Q.: On Marcinkiewicz integral with homogeneous kernel. J. Math. Anal. Appl. 245, 471-488 (2000)

4. Coifman, R., Meyer, Y.: On commutators of singular integrals and bilinear singular integrals. Transl. Am. Math. Soc. 212, 315-331 (1975)

5. Coifman, R., Meyer, Y.: Commutateurs d'inteégrales singuliéres et opérateurs multilinéaires. Ann. Inst. Fourier (Grenoble) 28, 177-202 (1979)

6. Christ, M., Journé, J:: Polynomial growth estimates for multilinear singular integral operators. Acta Math. 159, 51-80 (1987)

7. Kenig, C., Stein, E.: Multilinear estimates and fractional integration. Math. Res. Lett. 6, 1-15 (1999)

8. Grafakos, L., Torres, R.: Multilinear Calderón-Zygmund theory. Adv. Math. 165, 124-164 (2002)

9. Grafakos, L., Torres, R.: Maximal operator and weighted norm inequalities for multilinear singular integrals. Indiana Univ. Math. J. 51, 1261-1276 (2002)

10. Lerner, A., Ombrosi, S., Pérez, C., Torres, R., Trujillo-González, R.: New maximal functions and multiple weights for the multilinear Calderón-Zygmund theory. Adv. Math. 220, 1222-1264 (2009)

11. Chen, X., Xue, Q., Yabuta, K.: On multilinear Littlewood-Paley operators. Nonlinear Anal. 115, 25-40 (2015)

12. Xue, Q., Yabuta, K.: The existence and boundedness of multilinear Marcinkiewicz integrals on Companato spaces (2015) arXiv:1512.00663

13. Yang, D.: The central Campanato spaces and its application. J. Approx. Theory Appl. 10, $85-99$ (1994)

14. Lu, S., Yang, D.: Some new Hardy spaces on locally compact Vilenkin groups. Colloq. Math. 65, 117-138 (1993)

\section{Submit your manuscript to a SpringerOpen ${ }^{\circ}$ journal and benefit from:}

- Convenient online submission

- Rigorous peer review

- Open access: articles freely available online

- High visibility within the field

- Retaining the copyright to your article

Submit your next manuscript at $\boldsymbol{~ s p r i n g e r o p e n . c o m ~}$ 